\title{
Influence of Bubble-point Pressure on the Gas Formation in an Oil Reservoir Under Water Injection
}

\author{
Antonio Fernando Britto ${ }^{1, *}$, Ivan Costa da Cunha Lima ${ }^{1}$, Andre Telles da Cunha Lima ${ }^{1,2}$ \\ ${ }^{1}$ Post-graduation Department, University Center SENAI-Cimatec, Salvador, Brazil \\ ${ }^{2}$ Physics Institute, Federal University of Bahia, Salvador, Brazil

\section{Email address:} \\ ffbritto@hotmail.com (A. F. Britto), ivandacunhalima@gmail.com (I. C. da C. Lima), at.cunhalima@ufba.br (A. T. da C. Lima) \\ *Corresponding author
}

\section{To cite this article:}

Antonio Fernando Britto, Ivan Costa da Cunha Lima, Andre Telles da Cunha Lima. Influence of Bubble-point Pressure on the Gas Formation in an Oil Reservoir Under Water Injection. American Journal of Chemical Engineering. Vol. 9, No. 4, 2021, pp. 101-111. doi: 10.11648/j.ajche.20210904.14

Received: June 6, 2021; Accepted: June 24, 2021; Published: August 31, 2021

\begin{abstract}
To evaluate the oil recovering in a reservoir producing at the bubble-point pressure, we performed numerical simulations using a sandbox model and a black oil approach for the reservoir, and the tool-kit CFD software OpenFOAM. A new solver treats the three-phase dynamics of the oil-water-gas in the reservoir. The calculation includes four cases with different pressures of the injection and production wells to explore the free gas formation. Our results show that even keeping constant the pressure unbalance between the injection and production wells, we observe different dynamics. There is no gas formation and a typical production profile results if the bottom-hole pressure is just above the bubble-point in the injection and production wells. In case only the production well bottom-hole pressure is just below the bubble-point, we see no gas formation near the injection well and oscillatory gas formation around the production well. We see a triphasic flow along with the whole domain if both bottom-hole pressures are just below the bubble-point. However, if the bottom-hole pressure in both wells goes further below, the gas flow rate no more oscillates and the gas formation becomes continuous. We have also treated a special case to analyze the influence of gravity on the triphasic flow. Here we observed the gravity segregation to be not significant.
\end{abstract}

Keywords: Reservoir Simulation, Three-phase Flow, Porous Media, Computational Fluid Dynamics

\section{Introduction}

The sandbox model [1], allows for easily recognizing the concepts of geometric similarity, kinematic similarity, and dynamic similarity. We used the CFD software OpenFOAM [2] which is based on the finite volume method [3] where the main variables here are the pressure and the saturation of the wetting fluid, calculated by the implicit pressure and explicit saturation (IMPES) method [4]. The water pressure is the implicit variable and water saturation is the explicit variable. The model used for representing the oil was the black-oil model [5]. In this work, we selected the three-phase permeabilities model [6]. The capillary pressure, is calculated by empirical correlations [4]. Many of the technological effort were dedicated to remove the oil still in place. The change of the mobility ratio between water and oil has been pursued as a response to economic demands. Many of these efforts were dedicated to improve the oil secondary recovery in what is called Chemical Enhanced Oil Recovery (CEOR) [7].

For the last few decades many works dedicated to developing surfactants and polymers as agents for CEOR $[8,9,10]$. Some others are proposing Microbial Enhanced Oil Recovery (MEOR) [11] and in more recent developments the usage of nanoparticles has been the subject of extensive works [7, 12-16]. Most of them are based on laboratory experiments.

Under secondary recovery, some artificial lift method should be in place. With the continued reservoir depletion, it reaches the oil bubble point, meaning that gas will evolve from the oil phase. Gas and oil will get into the well bore and with the water influx, three-phase will flow into the equipment that will lift the fluids. This requires attention because some artificial lift methods like an electrical submersible pump (ESP) or progressive cavity pump (PCP) have a poor performance by handling free gas [17]. Delaying the production of gas and 
water is essentially the controlling factors in maximizing the fields ultimate oil recovery [18]. Many investigations have been made about the flow regimes where we have gas and liquid flowing simultaneously in tubes and pipes. In Gilbert [19] we have a description of the most accepted flow pattern regimes and in Duns [20] we have a report that rely upon empirical correlations and apply to critical flows at wells tubing. In more recent work by keeping the same approach, Ghareeb [21] proposed improvements for these correlations and mentions that reliable correlations for two-phase are limited and for multi-phase are rare. Because of the low Reynolds number, critical flows cannot be expected in porous media.

In this paper, we suggest how the reservoir simulation can help in the field development. In Section 2 we give a brief review of the porous media for a three-phase model, present the parameters used in the sandbox model, and the parameters for the black-oil model. We also present the equations implemented by the solver in the OpenFOAM software. Section 3 presents the results of the numerical simulations, comparing expected performances at different bottom-hole pressures above and below the oil bubble point. Several results compare the flow behavior by lowering the production bottomhole pressure. Discussing the consequences of that behavior, are presented in Section 3.

\section{Theoretical Developments}

\subsection{The Porous Media Model}

The equation governing the single-phase flow in a porous media is [1]:

$$
\frac{\partial(\rho \phi)}{\partial t}=\frac{(\rho K)}{\mu} \nabla \cdot(\nabla p-\rho g \nabla z)+q .
$$

Here $K$ is the permeability of the medium, $\nabla p$ the pressure gradient, $g$ is the gravity and $\nabla z$ the gradient of $z$ coordinate [1]. It results from mass conservation and the Darcy?s equation:

$$
\mathbf{u}=\frac{-K}{\mu}(\nabla p-\rho g \nabla z)
$$

Here $q$ is a source or a sink, and $\mathbf{u}$ is the velocity field. In triphasic flows, the saturations of the fluids correspond to the volume percentage of each fluid in the total porous volume. Thus, if $S_{w}$ is the percentage of water and $S_{o}$ the volume percentage of oil, and $S_{g}$ the volume percentage of gas, we have:

$$
S_{w}+S_{o}+S_{g}=1 .
$$

In a previous work [22] we modelled the flow in the reservoir by that of a black-oil [5] in a sandbox. We used the expressions of Delshad [6] and Chen [4] for the relative permeabilities and capillary pressures. The relative permeabilities equations developed by Delshad [6] are based on the two-phase data of Corey. The results we obtained were more consistent when compared with those in Refs. [23, 24, 25 ] give correlations for capillary pressure and permeabilities based on experimental data.

The following parameters describe the black-oil model [5]:

$$
\begin{gathered}
B_{o}=\frac{\left(V_{o}+V_{d g}\right)_{R C}}{\left(V_{o}\right)_{S T C}}, \\
B_{w}=\frac{\left(V_{w}\right)_{R C}}{\left(V_{w}\right)_{S T C}}, \\
B_{g}=\frac{\left(V_{g}\right)_{R C}}{\left(V_{g}\right)_{S T C}}, \\
R_{s}=\left(\frac{V_{d g}}{V_{o}}\right)_{S T C} .
\end{gathered}
$$

In these equations $B_{o}, B_{w}$ and $B_{g}$ are the formation factors referred to standard conditions $(S T C)$ and reservoir conditions $(R C)$ respectively. $V$ corresponds to the volume and the $d g$ subscript means the dissolved gas.The proposed correlations rely on the following data: (1) stock-tank oil gravity, ${ }^{\circ} A P I$; (2) first-stage separator gas specific gravity; (3) solution gasoil ratio at bubble point pressure, in standard cubic feet per stock-tank barrels (SCF/STB); (4) reservoir temperature, and (5) selected values of reservoir pressure. Equations (4 - 7) are calculated at each time step for all meshes. The densities of the three phases at reservoir conditions are related to densities at STC:

$$
\begin{gathered}
\rho_{o}=\frac{1}{B_{o}}\left(\rho_{o S T C}+R_{s} \rho_{g S T C}\right), \\
\rho_{w}=\frac{1}{B_{w}} \rho_{w S T C}, \\
\rho_{g}=\frac{1}{B_{g}} \rho_{g S T C} .
\end{gathered}
$$

The oil density may also be expressed as:

$$
\rho_{o}=\bar{\rho}_{o}+\bar{\rho}_{d g S T C} .
$$

In Eq. (11) $\bar{\rho}_{o}$ and $\bar{\rho}_{d g}$ are densities of the two components:

$$
\overline{\rho_{o}}=\frac{1}{B_{o}} \rho_{o S T C} \quad \text { and } \quad \overline{\rho_{g}}=\frac{R_{s}}{B_{o}} \rho_{g S T C}
$$

Figures 1(a), 1(b), 2(a) and 2(b) show the formations factors $B_{o}, B_{g}$, the densities for oil and gas phases, the gas formation ratio and the compressibility factor $Z$ as functions of the pressure. We used the correlations for the black-oil as in Ref. [5], and the compressibility factor according to Ref. [26]. Our results are in good agreement with the data published in Ref. [27], [28] and [29].

Equation (1) can be specific for each phase:

$$
-\nabla \cdot\left(\frac{1}{B_{o}} \mathbf{u}_{o}\right)=\frac{\partial}{\partial t}\left(\frac{1}{B_{o}} \phi S_{o}\right)+q_{o},
$$




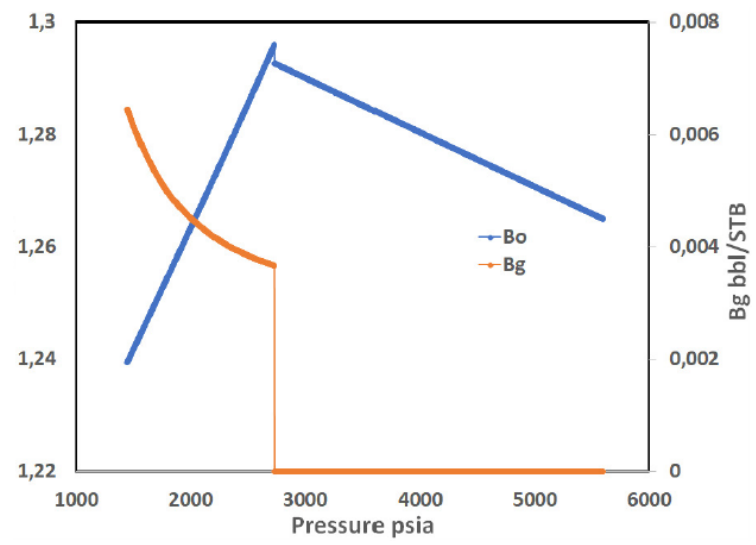

(a)

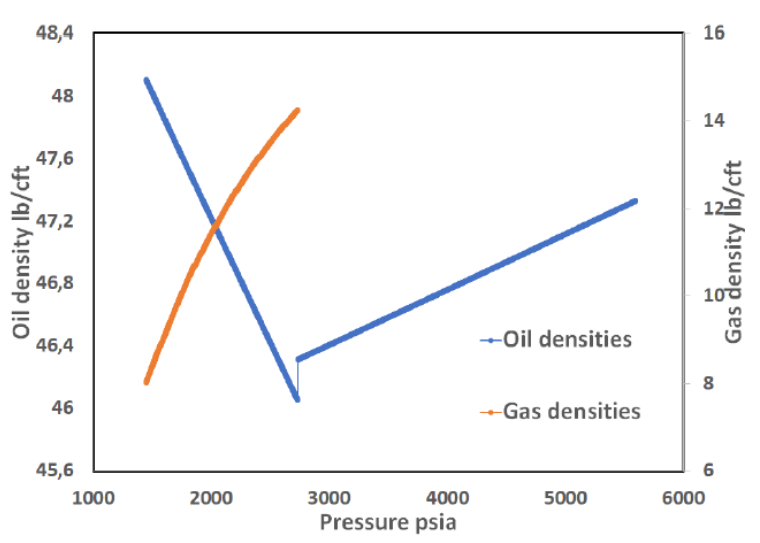

(b)

Figure 1. (a)Formations factors $B_{o}$ and $B_{g}$ and $(b)$ densities for oil and gas phases.

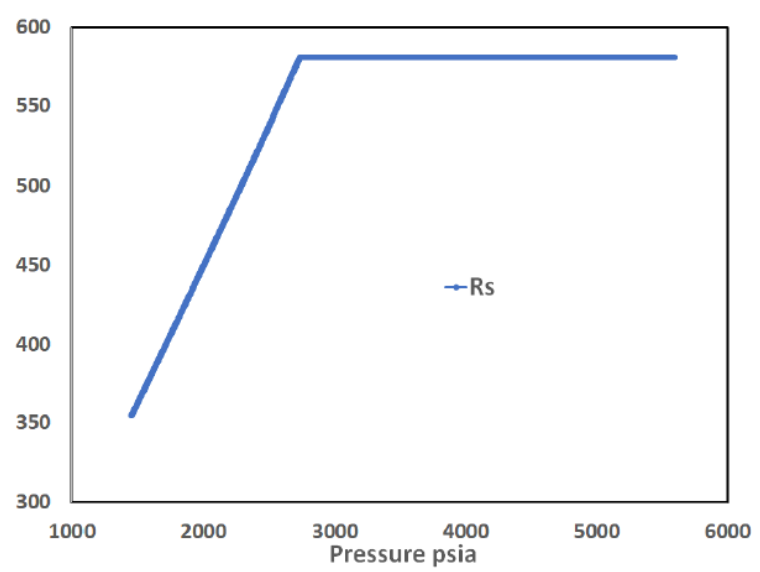

(a)

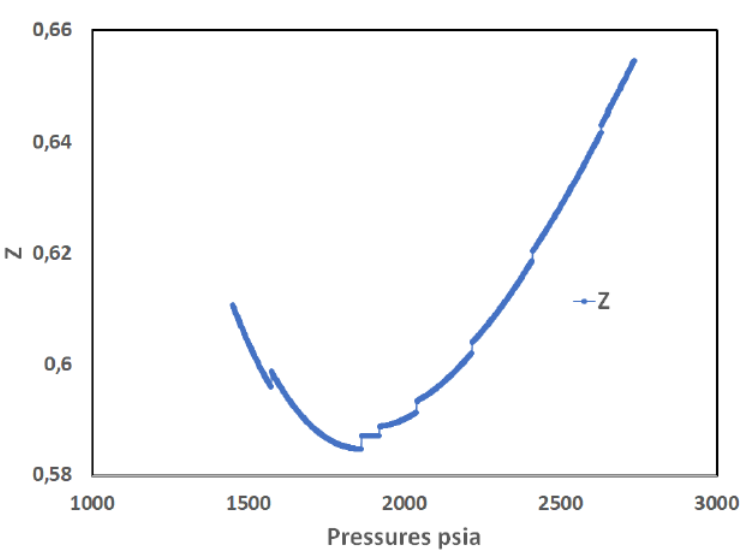

(b)

Figure 2. (a) Gas formation ratio and (b)compressibility factor $Z$.

$$
\begin{gathered}
-\nabla \cdot\left(\frac{1}{B_{w}} \mathbf{u}_{w}\right)=\frac{\partial}{\partial t}\left(\frac{1}{B_{w}} \phi S_{w}\right)+q_{w} \\
-\nabla \cdot\left(\frac{R_{s}}{B o}+\frac{1}{B_{g}} \mathbf{u}_{g}\right)=\frac{\partial}{\partial t}\left(\phi\left(\frac{R_{s}}{B_{o}} S_{o}+\frac{1}{B_{g}} S_{g}\right)\right)+q_{f g}+R_{s} q_{o} .
\end{gathered}
$$

Notice that gas is present as free gas $f g$, and in oil phases as dissolved gas $d g$. We define the parameters $\lambda$ and $\gamma$ as the mobility and specific weight. Also, $p_{\text {cow }}$ is the oil-water capillary pressure and $p_{\operatorname{cog}}$ is that for oil-gas. Then we coded the following equations into OpenFOAM:

$$
\begin{gathered}
K\left(\nabla \cdot\left(\lambda_{w}\left(\nabla p_{w}-\gamma_{w} \nabla z\right)\right)+\frac{B_{o}}{B_{w}} \nabla \cdot\left(\lambda _ { o } \left(\nabla p_{w}-\nabla p_{c o w}-\right.\right.\right. \\
\left.\left.\left.\gamma_{o} \nabla z\right)\right)+\frac{B_{g}}{B_{w}} \nabla \cdot\left(\lambda_{g}\left(\nabla p_{w}-\nabla p_{c o g}-\gamma_{g} \nabla z\right)\right)\right)=q_{w}+\frac{B_{o}}{B_{w}} q_{o}+\frac{B_{g}}{B_{w}} q_{f g} \\
B_{w} \frac{\partial S_{w}}{\partial t}-\nabla \cdot\left(\lambda_{w} K\left(\nabla p_{w}-\gamma_{w} \nabla z\right)\right)=q_{w} \\
B_{o} \frac{\partial S_{o}}{\partial t}-\nabla \cdot\left(\lambda_{o} K\left(\nabla p_{w}-\nabla p_{c o w}-\gamma_{w} \nabla z\right)\right)=\frac{B_{o}}{B_{w}} q_{o}
\end{gathered}
$$




\subsection{Model Scale}

The equations governing the dynamics of the flow can be written in a dimensionless form [23]. In the case of a three phase flow, with the proper scales for length $\left(L_{0}\right)$, time $\left(T_{0}\right)$, pressure unbalance $\left(\Delta p_{0}\right)$, density $\left(\rho_{0}\right)$, permeability $\left(K_{0}\right)$, and viscosity $\left(\mu_{0}\right)$, we arrived to two factor determining dynamic similarity for geometrically similar reservoir and model:

$$
N_{r 1}=\frac{T_{0} K_{0}}{\mu_{0} L_{0}} \frac{\Delta p_{0}}{L_{0}}, \quad N_{r 2}=\frac{T_{0} K_{0} \rho_{0} g}{\mu_{0} L_{0}}
$$

Table 1 shows the values of these parameters for a typical reservoir:

Table 1. Real reservoir parameters.

\begin{tabular}{ll}
\hline Parameter & Value \\
\hline Water density & $1000 \mathrm{~kg} / \mathrm{m}^{3}$ \\
Oil density & $800 \mathrm{~kg} / \mathrm{m}^{3}$ \\
Water Viscosity & $0.0025 \mathrm{~kg} / \mathrm{ms}$ \\
Oil Viscosity & $0.004 \mathrm{~kg} / \mathrm{ms}$ \\
Gravity & $9.81 \mathrm{~m} / \mathrm{s}^{2}$ \\
Absolute Permeabillity & $1000 \mathrm{md}$ \\
Porosity & 0.2 \\
Initial Water Saturation, $S_{w i}$ & 0.2 \\
Residual Water Saturation, $S_{r w}$ & 0.15 \\
Residual Oil Saturation, $S_{r o}$ & 0.81 \\
\hline
\end{tabular}

In our simulations we examined two relevant control variables: the running time and the accuracy of the processing. We considered a sandbox model in the shape of a cube with dimensions $30 \mathrm{~m} \times 30 \mathrm{~m} \times 30 \mathrm{~m}$. The injection and production wells were at the same level at $17 \mathrm{~m}$ from the bottom. After some test runs, we concluded we should split the entire domain into $80 \times 80 \times 50$ cells, i.e., 320,000. This was necessary to have a consistent material balance and a negligible change in results when the cells number increases. The time step was $0.001 \mathrm{~s}$. In order to plot the dynamic variables, we used the parameter simulations time units which corresponds to $10^{3}$ time steps. Using the parameters in Table $1, L_{0}=30 \mathrm{~m}$ and considering the result of the time for breakthrough as $T_{0}=60 \mathrm{~s}, \Delta p=300 \mathrm{psi}$, we obtain $N_{r 1}=0.34$, and $N_{r 2}=$ 4. $\times 10^{-6}$. These two factors convert our results calculated in our sandbox into a dynamically and geometrically similar reservoir. As initial conditions, we assumed $S_{w}=0.2$ and $p_{w}=2.3 \times 10^{7}$ pascal.

\subsection{Solver for the Three Phase Flow}

We developed a solver for the system of coupled differential equations, Eqs (16 - 18), and we used the CFD software OpenFOAM [2] to programming the solver code. OpenFOAM CFD uses classes in $C^{++}$language that are developed aiming at this application, where a system of coupled differential equations needs to be solved numerically. At each time step, all pressure-dependent properties are updated for the entire domain. For the mathematical resolution, we selected the IMPES (Implicit pressure explicit saturation) [30] method developed by Sheldon [31] and Stone [32], to obtain better stability without increasing the computational complexity. So, in equation 16 the water pressure is the implicit variable, and water and oil saturations are the explicit variables. Table 2 shows a sketch of how the method works in our simulations.

Table 2. IMPES method.

\begin{tabular}{lllll}
\hline & $\mathbf{t}$ & $\mathbf{t}+\Delta \mathbf{t}$ & & $\mathrm{t}+n \Delta \mathrm{t}$ \\
\hline$p_{w}^{0}$ & $p_{w}^{t}\left(S_{w}^{0}\right)$ & $p_{w}^{t+\Delta t}\left(S_{w}^{t}\right)$ & $\ldots$ & $p_{w}^{t+n \Delta t}\left(S_{w}^{t+(n-1) \Delta t}\right)$ \\
$S_{w}^{0}$ & $S_{w}^{t}\left(p_{w}^{t}\right)$ & $S_{w}^{t+\Delta t}\left(p_{w}^{t+\Delta t}\right)$ & $\ldots$ & $S_{w}^{t+n \Delta t}\left(p_{w}^{t+n} \Delta t\right)$ \\
\hline
\end{tabular}

The equations in $C^{++}$language and using OpenFOAM classes are:

$$
\begin{aligned}
& \text { fvScalarMatrix pEqn } \\
& \text { - fvm::laplacian }\left((\text { lambdaw + lambdao }) *\left(\frac{B o}{B w}\right)+\right.\text { lambdag* } \\
& \left.\left(\frac{B g}{B w}\right) * K, p w\right)- \text { fvc::laplacian }\left(\left(\frac{B o}{B w}\right) * \text { lambdao }+\left(\frac{B g}{B w}\right)\right) * \\
& (\text { lambdag } * K, P c o w)-\text { fvc::laplacian }\left(\left(\frac{B g}{B w}\right) * \operatorname{lambdag} * K, P c o g\right) \\
& + \text { fvc::div }\left(\text { gamaw } * \text { lambdaw }+\left(\frac{B o}{B w}\right) * \text { gamao*lambdao }+\right. \\
& \left.\left.\left.\left(\frac{B g}{B w}\right) * \text { gamag } * \text { lambdag } * K\right)\right) \& g\right) \\
& ) ;
\end{aligned}
$$




\section{fvScalarMatrix sEqn1}

$$
\begin{aligned}
& \text { fvm::ddt }\left(\frac{\text { porosity }}{(\mathrm{Bw} * \text { intervals })}, \mathrm{sw}\right) \\
& \text { - fvc::laplacian }(\text { lambdaw } * \mathrm{~K}, \mathrm{pw})+ \\
& \text { fvc::div }((\text { gamaw } * \text { lambdaw } * \mathrm{~K}) \& \mathrm{~g}) \\
& ) ;
\end{aligned}
$$

\section{fvScalarMatrix sEqn2}

$$
\begin{aligned}
& \text { fvm::ddt }\left(\frac{\text { porosity }}{(\text { Bo } * \text { intervals })}, \text { so }\right) \\
& \text { - fvc::laplacian(lambdao } * \mathrm{~K}, \mathrm{pw})- \\
& \text { fvc::laplacian(lambdao } * \mathrm{~K}, \mathrm{Pcow})+ \\
& \text { fvc::div(gamao } * \text { lambdao*K \& g) } \\
& \text { ); }
\end{aligned}
$$

\section{Results}

The simulations were performed in four cases, where we keep always the same $\Delta p$ of $6.9 \times 10^{5}$ pascal (100 psi) between the injection and production wells. All displayed information refers to reservoir conditions. We considered that the reservoir is under water influx, producing under artificial water drive mechanism and keeping hydraulic control. According to correlations provided by McCain [5], we assumed a typical black-oil of $35^{0} A P I$, gas density at separator $\gamma_{g s p}$ of 0.82 , $R_{S P}$ of $500 \mathrm{ft}^{3} / \mathrm{bbl}$ and a reservoir temperature of $90^{\circ} \mathrm{F}$, with an estimated bubble point of $1.89 \times 10^{7}$ pascal. The wellbore pressures of injection and production wells were set up according to table 3 .

Table 3. Well bore pressures in Pascal.

\begin{tabular}{lll}
\hline Case number & Injection well & Production well \\
\hline 1 & $1.96 \times 10^{7}$ & $1.89 \times 10^{7}$ \\
2 & $1.92 \times 10^{7}$ & $1.85 \times 10^{7}$ \\
3 & $1.7 \times 10^{7}$ & $1.63 \times 10^{7}$ \\
4 & $1.2 \times 10^{7}$ & $1.13 \times 10^{7}$ \\
\hline
\end{tabular}

In case 1 , both injection and production wells have their bottom pressure above the bubble point. Figures 3(a) and 3(b) show a typical production profile from a reservoir under water drive recovery mechanism. No free gas develops from the oil phase. After around 32 time units, water breaks into the production well characterizing the breakthrough. Then the oil production declines.
In case 2 we have the reservoir only partially in three phases. This is because the bottom-hole pressure at the production well is slightly below the bubble point of the oil, creating a low-pressure zone around it. With the gas releasing, it can produce instantaneously more oil than in case 1 because we are adding the gas expansion driver to the water driver recovery mechanism. Figures 4(a) and 4(b) show water injection and oil, water, and gas production. The figures are showing the time interval for the reservoir, including the major events along with their lifetime. However, the fast oscillatory behavior of the gas phase makes the other flows vary in such a way that actually what we can see is the colored range in the graphics.

In case 3, the whole reservoir is experiencing three phases. The bottom-hole pressure at the injection well is also slightly below the oil bubble point, meaning that all three phases coexist in the whole reservoir. More gas develops from the oil than in case 2 and increases the gas expansion driver recovery mechanism and it can produce more oil, in consequence. However, we can observe that the flow rates have more intense variations, coloring a larger part of the chart than in case 2 . Figures 5(a) and 5(b) show the water injection and oil, water, and gas production.

In case 4 , the pressures were further lowered, rendering more gas in such a way that we cannot observe flow rate variations, and in fact, the gas flow rate becomes continuous. This case exhibits lower production of oil and gas compared to cases 2 and 3. This happens because, at this pressure level, most of the gases are already available since the beginning of reservoir production and the gas expansion driver does not contribute much to the mechanism of recovery. Figures 6(a) and 6(b) show the water injection and oil, water, and gas production.

Figures 7(a) to 8(b) show the variations observed in Figs. 4(a) and 5(a) as we expand the time scale interval between 10.00 and 10.35 time units. Upon reaching the oil bubble point, we see in Figure 7(a) that the gas comes out from the liquid, and after some time the gas bubbles collapse. It is important to note that there are several variables involved in this phenomenon, each one having its dynamics. Before reaching the breakthrough, some water gets already into the production well, since it was present since the beginning in the reservoir, and it has higher mobility than the oil. However, the water does not come out at a constant rate, and this we can see in Figure 7(b) where the water saturation declines. In this scenario, the oil rate increases, and so does the oilwater capillary pressure. Once the bubble point is reached, gas develops, but the occupied volume by the developed gas is greater than that of the oil. Under these conditions, there is an increase in the gas-oil capillary pressure contributing to a global effect of a slow pressure increase. The outcome is that the bubble point is surpassed and the bubbles of developed gas collapse. We have compared the oil accumulated productions for the cases studied. Figure 9 confirms that under combined driver recovery mechanism, we can recover more oil at the referred time interval of this study. 


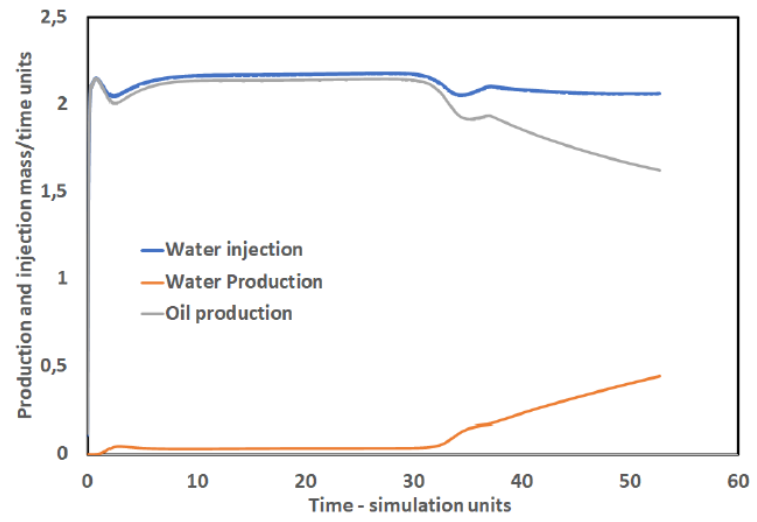

(a)

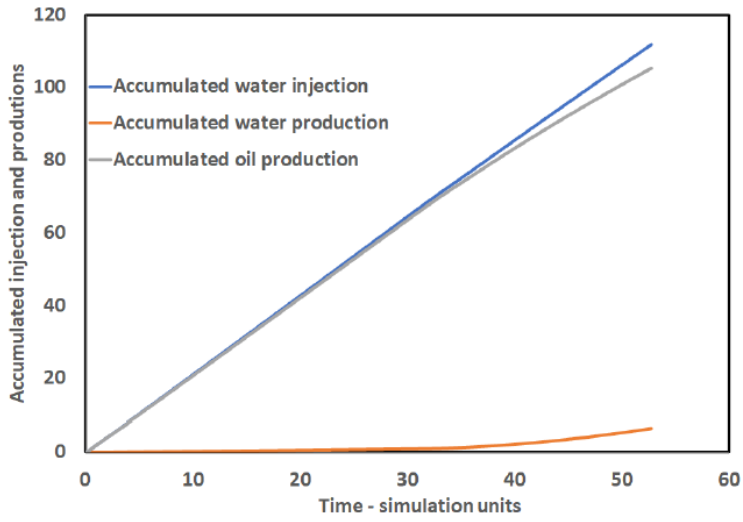

(b)

Figure 3. Instataneous (a) and accumulated $(b)$ water injection and oil and water productions for injection well pressure of $1.96 \times 10^{7}$ pascal and production well pressure $1.89 \times 10^{7}$ pascal producing no free gas.

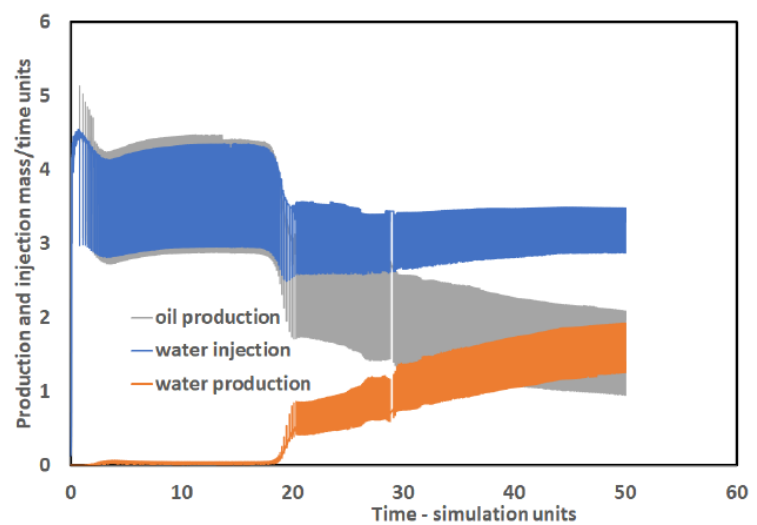

(a)

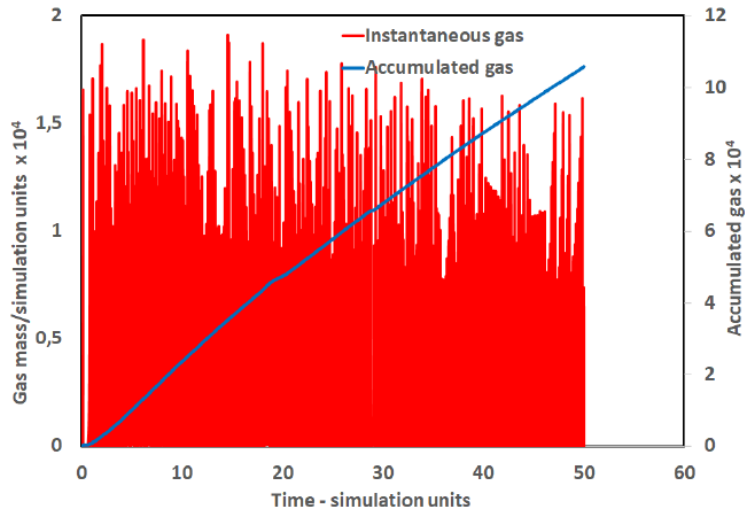

(b)

Figure 4. (a) Oscillating water injection and water and oil production, (b) oscilatting gas production for injection well pressure of $1.92 \times 10^{7}$ pascal and production well pressure $1.85 \times 10^{7}$ pascal. Production well bottom-hole pressure slightly below oil buble point.

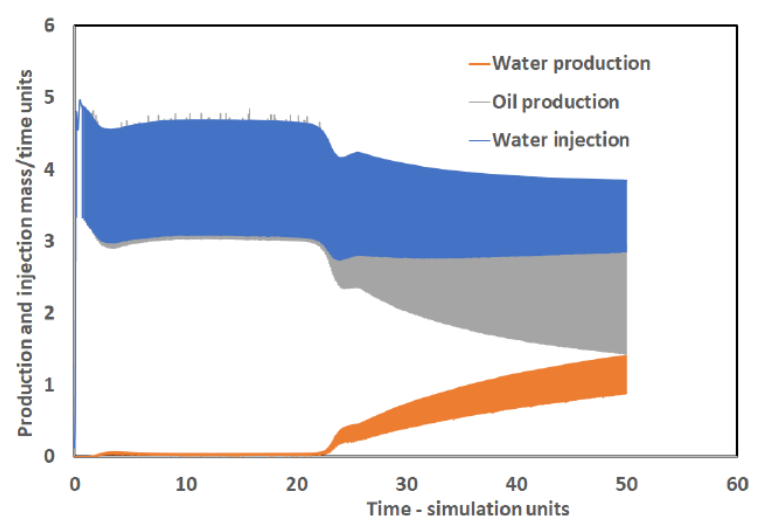

(a)

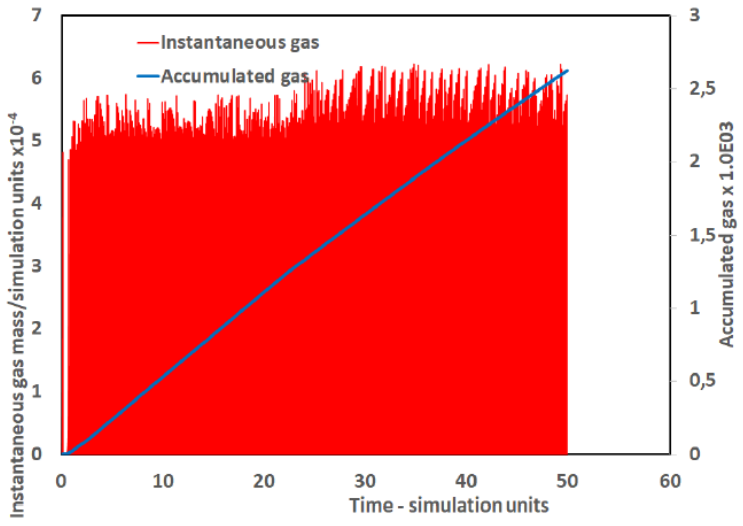

(b)

Figure 5. (a) Oscillating water injection and water and oil production, (b) oscilatting gas production for injection well pressure of $1.7 \times 10^{7}$ pascal and production well pressure $1.63 \times 10^{7}$ pascal. Well production bottom-hole pressure slightly below oil buble point. 


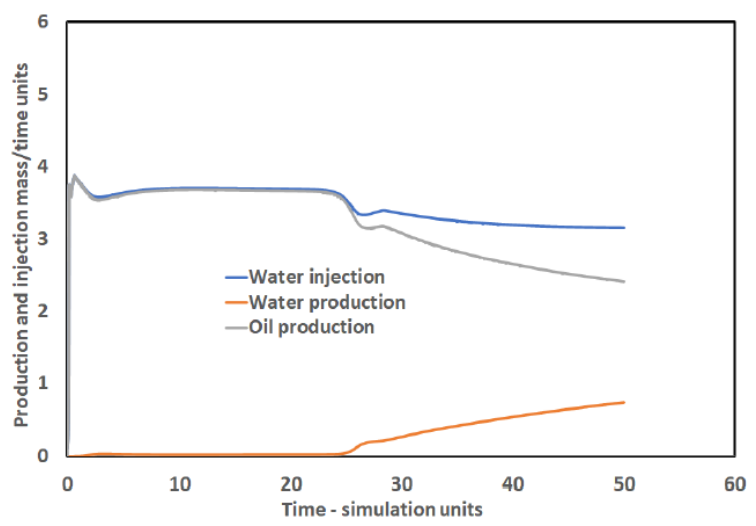

(a)

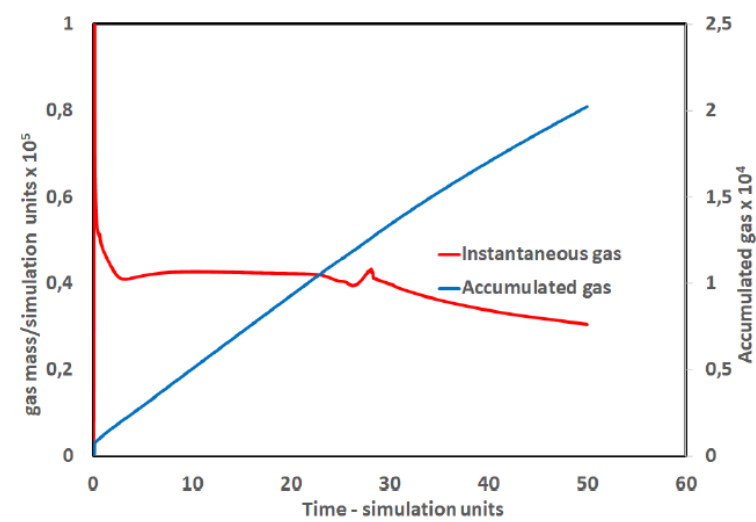

(b)

Figure 6. (a) Oscillating water injection and water and oil production, (b) oscilatting gas production for injection well pressure of $1.2 \times 10^{7}$ pascal and production well pressure $1.13 \times 10^{7}$ pascal. Well production bottom-hole pressure further below oil buble point causing continuous gas production.

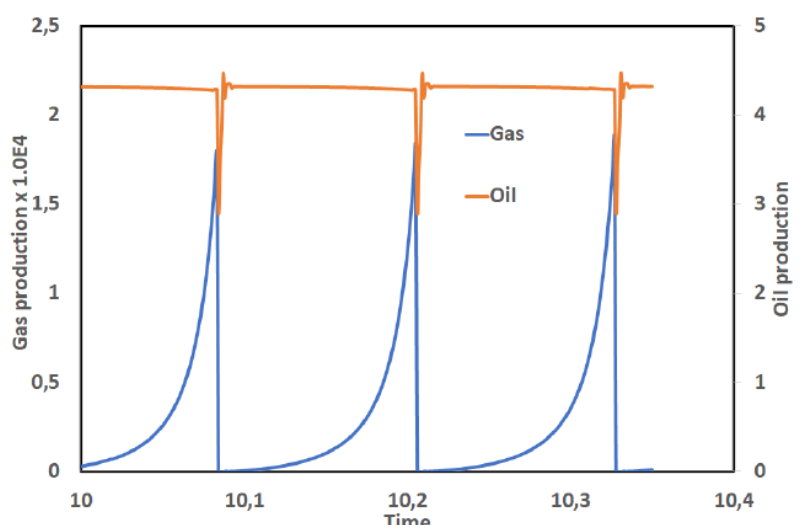

(a)

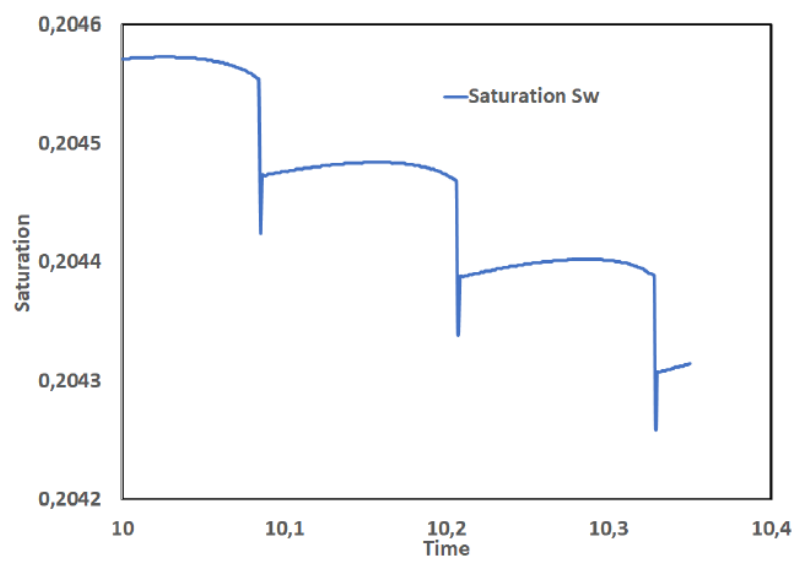

(b)

Figure 7. Oil and gas productions and water saturation between 10 and 10,35 time units.

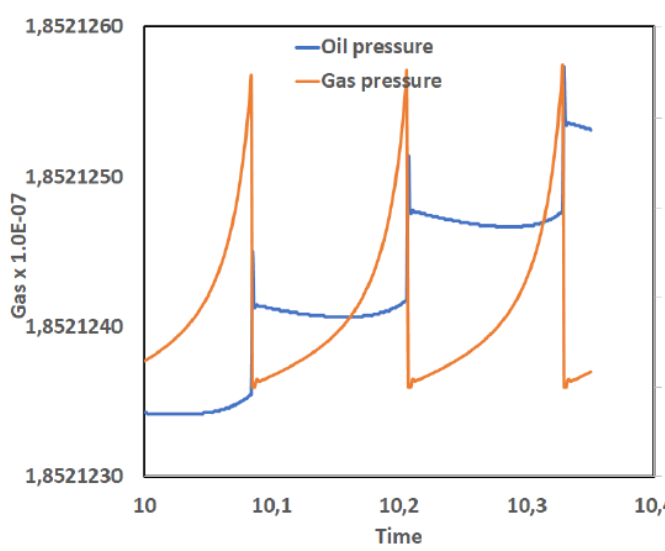

(a)

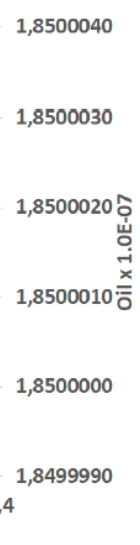

21270
21255
$\overline{0}$
21240
21225
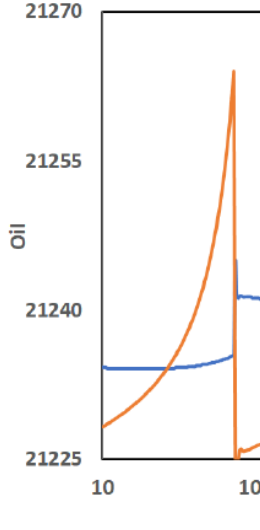

Figure 8. Pressures and capillary pressures between 10 and 10,35 time units. 


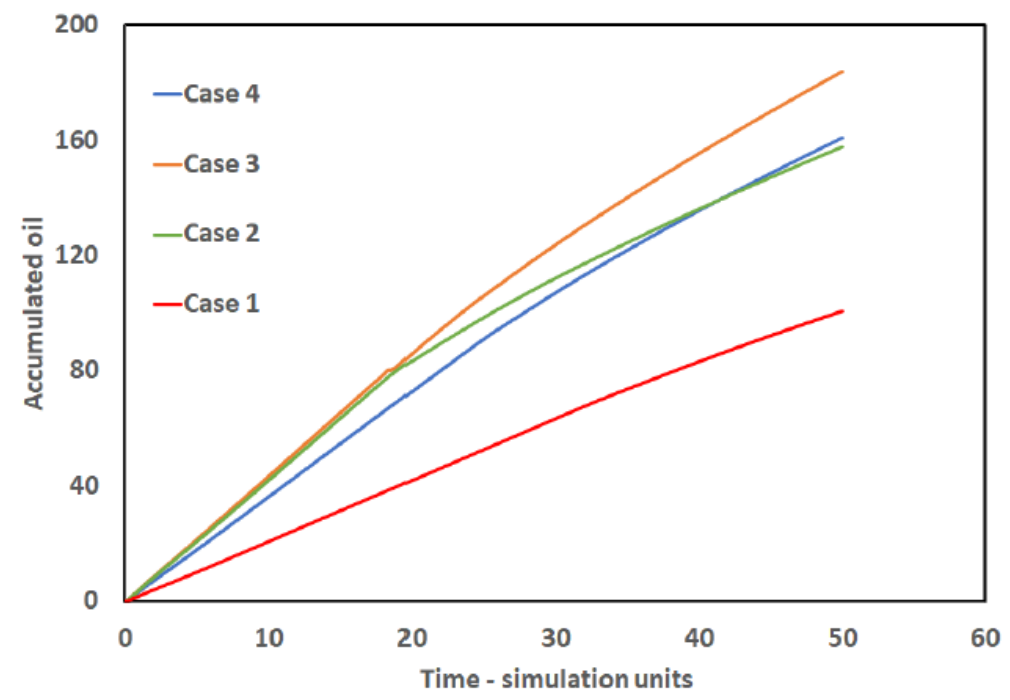

Figure 9. Accumulated oil recoveries.

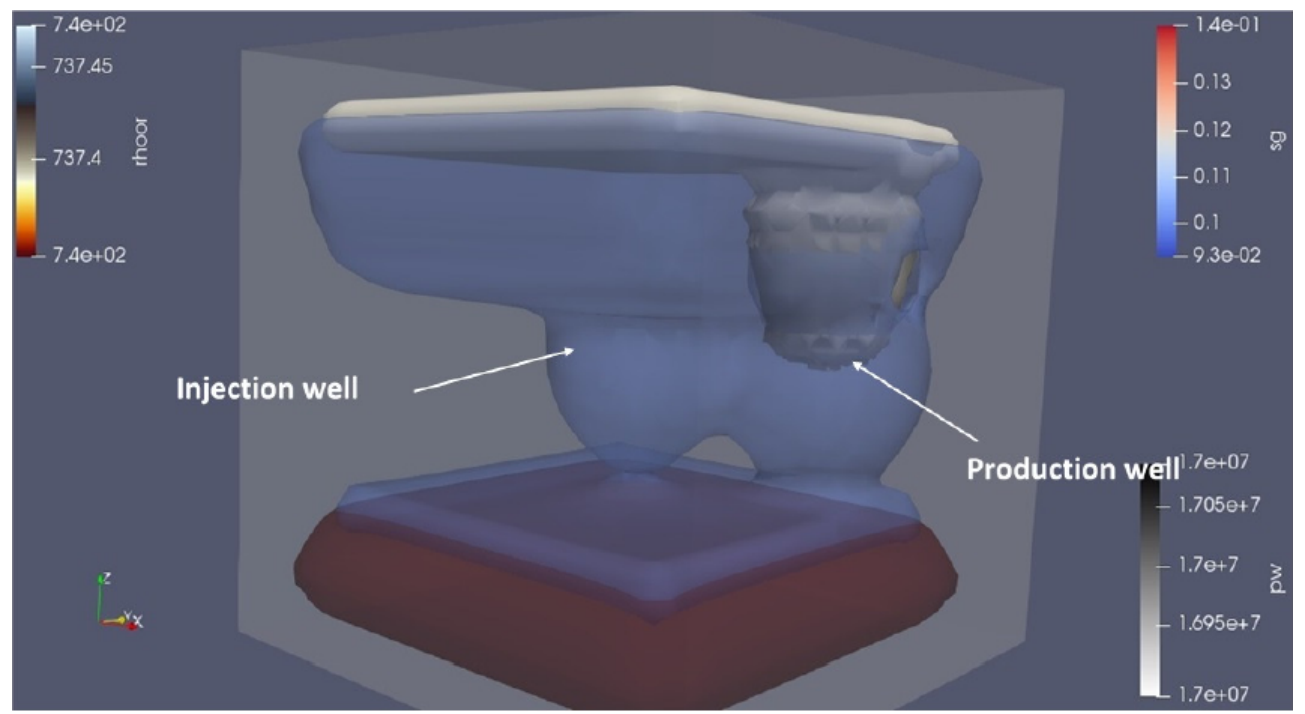

Figure 10. Three dimensional snapshot displaying the gravity segregation after 500 time simulations units.

A question that could be raised concerns how important is the gravity segregation in the recovery process. Aiming that point we performed a special simulation controlling the pressures of the injection and producing wells in a such way that no water inflow nor production could be obtained. This was achieved by adjusting the bottom-hole pressure of the injection and production wells in such a way to have the same value at those cells containing the wells. We set the boundary conditions defining the reservoir by having an uniform initial distribution of gas, oil, and water. We set the reservoir pressure below the oil bubble point. From Eqs (16 -18), the saturation of water, oil and gas must change with pressure. With no input and output flow, it takes longer for the gravitational segregation to take effect and justify the change in the sandbox appearance, starting from uniform to that one we have got in figure 10 . After 500 simulation time units, the gravity segregation of approximately $5 \%$ could be observed. Figure 10 shows a three- dimensions snapshot of the final appearance of our sandbox model where we can see in red the water, in white the gas, and the oil in blue. This result explains why in those cases we could not observe gravitational segregation, once the simulations performed in the four cases took only 50 simulation time units.

At the time of discovery, oil reservoirs may present a gas cap (saturated reservoir) or may not (undersaturated reservoir). The undersaturated reservoir pressure is above the oil bubble point, and only the oil phase is present. With the continued production, the pressure decreases and it may reach the oil bubble point. We expect the creation of an artificial gas cap by the process of gravity segregation. However, in the case we treated, it should take a very long time, and perhaps not much gravity segregation will occur during the reservoir lifetime. Craft [27] mentions that the free gas does not necessarily rise to form an artificial gas cap and should remain distributed throughout the reservoir as isolated bubbles. 


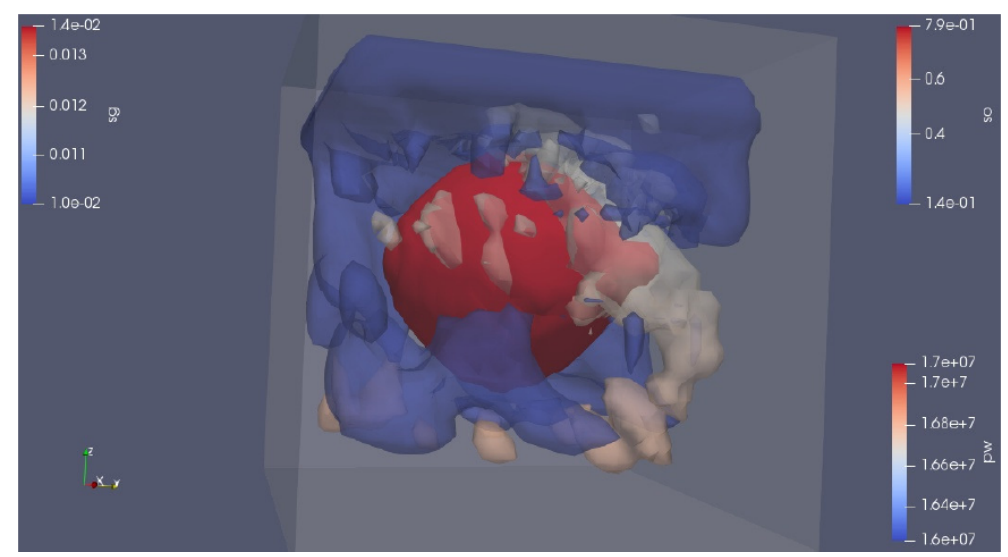

Figure 11. A snapshot of the three phase breakthrough. In red color, we see the spherical shape with the spout reaching the production well. In blue, we see the oil being displaced by the water-front. In light yellow, we see gas the formation around the production well.

Our simulations show that the water creates a spherical front that is deformed as it approaches the production well. We can see this in Figure 11. At the very beginning, the water spherical front is deformed by the effect of gravity. As the water-front approaches the producing well, it forms something like a water-spout. When the water-spout reaches the producing well, it establishes the breakthrough and reduces the oil recovery rate, because water is added to the produced fluids. In red color we see the moment where this spherical shape with the spout reaches the production well. The spout is always formed in the gradient pressure direction. In blue we see the oil being displaced by the water-front. Because the production well is a low-pressure zone, upon reaching the bubble point, gas develops from oil and flows through the well. In Figure 11 we can observe in light yellow the gas formation around the production well. The results suggest that the transition from the liquid phase to the liquid dominant phase with gas in the porous media may not be smooth, and we can expect pressure variations. We associate this with the fact that, once it reaches the bubble point, the gas phase comes out and the pressure response decreases. Once almost all gas is released, the gas production becomes continuous. However, at that point the gas expansion driver recovery mechanism is no longer significant. Satter [33] reports this phenomenon. Our results are also in good agreement with his conclusion about the gas production over the reservoir's lifetime. As stated by Clegg [17], it is very important to know the actual reservoir flow pattern, because it affects the calculation of the inflow performance relationship (IPR).

\section{Conclusions}

Most reservoir simulators are commercial packages. In this work, we used an open-source simulator and introduced a tool in such a way that we can add any model for the relative permeability and capillary pressure of the black-oil. This way anyone can freely configure and customize his/her model for fluid and rock properties. OpenFOAM CFD comprises a very large user community that is always committed to develop and deploy solutions for specific problems. This work provides a tool that may be further improved by aggregating new functionalities. As an open-source tool, the proposed solver can be adjusted and parameters customized, as well as the capillary pressure and relative permeabilities models can be fine-tuned, providing a better representation of the phenomena.

In the field's life, aiming at increasing the production, the employment of some artificial lift method can be beneficial. To realize the maximum potential from developing any oil or gas field, we must select the most economical artificial lift method. Also, misconceptions on the performance prediction of the reservoir may lead to oversized equipment for artificial lift [17]. When under-loaded, these types of equipment often exhibit poor efficiencies incurring increased energy costs. Even worse is when multiple changes in size or type are required in the installed lift equipment. For instance, an installation where we have an electrical submersible pump working as artificial lift equipment will not perform well if gas comes at significant rates. A similar problem will happen with the progressive cavity pump artificial lift equipment. A change to the artificial gas lift method will require enormous investments.

In the present simulations we could identify the formation of different phases inside the body of the reservoir, and how that formation is controlled by the bottom-hole pressures of the injection and the production wells, and not only by their pressure unbalance. Another point is that we could observe a transient phenomenon that happens since the very beginning of the gas formation. Depending on how much below the bubble point we are, we can observe this phenomenon no longer. The gas dynamic behavior affects the production profile of the field. The mapping onto real reservoirs, however, does not depend only on the parameters leading to dynamical similarity, but also on other questions like up-scaling and adjustments on the reservoir walls permeabilities. 


\section{Author's Contributions}

All authors contributed equally to this work.

\section{Availability of Data}

The data and the codes in OpenFOAM/C++ that were used and support the findings of this work are available from the corresponding author upon reasonable request.

\section{Acknowledgements}

Antonio Fernando Britto acknowledges INCT-GP for a fellowship DTI-C process number 380394/2020-0 from CNPq-Brazil.

\section{References}

[1] J. Bear, Dynamic of fluids in porous media. New York: Dover Publications, 1972.

[2] C. Greenshields, OpenFOAM Userguide. OpenFOAM Fundation LTD, 2018.

[3] H. K. Versteeg and W. Malalasekera, An introduction to computational fluid dynamics: the finite volume method. London, 2007: Pearson Education, 2007.

[4] Z. Chen, G. Huan, and Y. Ma, Computational Methods for Multiphase Flows in Porous Media. Phyladelphia, 2006: Society for Industrial and Applied Mathematics, 2006.

[5] W. D. McCain, J. P. Spivey, and C. P. Lenn, Petroleum Reservoir Fluid Properties Correlations. Tulsa, 2011: PennWell Corporation, 2011.

[6] M. Delshad and G. A. Pope, "Comparison of the threephase oil relative permeability models," Transport in Porous Media, vol. 4, no. 1, pp. 59“C83, 1989.

[7] J. A. Ali, K. Kolo, A. K. Manshad, and A. H. Mohammadi, "Recent advances in application of nanotechnology in chemical enhanced oil recovery: Effects of nanoparticles on wettability alteration, interfacial tension reduction, and flooding," Egyptian journal of petroleum, vol. 27, no. 4, pp. 1371“'C1383, 2018.

[8] D. J. Pye, "Improved secondary recovery by control of water mobility," Journal of Petroleum technology, vol. 16, no. 08, pp. $911^{*} \mathrm{C} 916,1964$.

[9] W. Gogarty, "Mobility control with polymer solutions," Society of Petroleum Engineers Journal, vol. 7, no. 02, pp. $161{ }^{*} \mathrm{C} 173,1967$.
[10] A. Abidin, T. Puspasari, and W. Nugroho, "Polymers for enhanced oil recovery technology," Procedia Chemistry, vol. 4, pp. 11“C $16,2012$.

[11] R. Khademolhosseini, A. Jafari, and M. Shabani, "Micro scale investigation of enhanced oil recovery using nano/bio materials," Procedia Materials Science, vol. 11, pp. $171{ }^{\circ} \mathrm{C} 175,2015$.

[12] A. Roustaei, S. Saffarzadeh, and M. Mohammadi, "An evaluation of modified silica nanoparticles efficiency in enhancing oil recovery of light and intermediate oil reservoirs," Egyptian Journal of Petroleum, vol. 22, no. 3, pp. 427“C433, 2013.

[13] C. Negin, S. Ali, and Q. Xie, "Application of nanotechnology for enhancing oil recovery. a review," Petroleum, vol. 2, no. 4, pp. 324“C333, 2016.

[14] H. Yousefvand and A. Jafari, "Enhanced oil recovery using polymer/nanosilica," Procedia Materials Science, vol. 11, pp. 565“C570, 2015.

[15] M. I. Youssif, R. M. El Maghraby, S. M. Saleh, and A. Elgibaly, "Silica nanofluid flooding for enhanced oil recovery in sandstone rocks," Egyptian Journal of Petroleum, vol. 27, no. 1, pp. $105^{\circ} \mathrm{C} 110,2018$.

[16] C. R. Miranda, L. S. d. Lara, and B. C. Tonetto, "Stability and mobility of functionalized silica nanoparticles for enhanced oil recovery applications," in SPE international oilfield nanotechnology conference and exhibition, pp. 1“C 11, Society of Petroleum Engineers, 2012.

[17] J. D. Clegg, Volume IV-Production Operations Engineering. Society of Petroleum Engineers, 2007.

[18] T. Ahmed and N. Meehan, Advanced Reservoir Management and Engineering. Amsterdan, 2012: Gulf Professional Publishing, 2012.

[19] W. Gilbert, "Flowing and gas-lift well performance," in Drilling and production practice, pp. 1"C23, American Petroleum Institute, 1954.

[20] H. Duns Jr and N. Ros, "Vertical flow of gas and liquid mixtures in wells," in 6th world petroleum congress, pp. 1“C16, World Petroleum Congress, 1963.

[21] M. Ghareeb and S. Elgaghah, "A new correlation for calculating wellhead production considering influences of temperature, gor, and water-cut for artificially lifted wells," in International Petroleum Technology Conference, pp. $\quad{ }^{\circ}{ }^{\circ} \mathrm{C} 10$, International Petroleum Technology Conference, 2007.

[22] A. Britto, C. Vivas, M. Almeida, I. da Cunha Lima, and A. da Cunha Lima, "Multiphase flow mobility impact on oil reservoir recovery: An open-source simulation," AIP Advances, vol. 10, no. 3, pp. 035032 1“C C10, 2020. 
[23] R. Brooks and A. Corey, "Hydrology properties of porous media.," Hydrology Papers, vol. 2, no. 3, pp. 10"C 23, 1964.

[24] D. D. Huang, M. M. Honarpour, and R. Al-Hussainy, “An improved model for relative permeability and capillary pressure incorporating wettability," in SCA, vol. 9718, pp. $7{ }^{*} \mathrm{C} 10,1997$.

[25] S. Skjaeveland, L. Siqveland, A. Kjosavik, W. Hammervold, G. Virnovsky, et al., "Capillary pressure correlation for mixed wet reservoirs," in SPE India Oil and Gas Conference and Exhibition, Society of Petroleum Engineers, 1998.

[26] P. Dranchuk, H. Abou-Kassem, et al., "Calculation of z factors for natural gases using equations of state," Journal of Canadian Petroleum Technology, vol. 14, no. 03, pp. $105{ }^{\circ} \mathrm{C} 108,1975$.

[27] R. E. Craft, B. C. Terry and J. B. Rogers, Applied Petroleum Reservoir Engineering. Englewoods Cliffs, 1991: Prentice-Hall, 2007.

[28] T. Ahmed, Reservoir Engineering Handbook. Burlington, 2010: Gulf Professional Publishing, 2010.

[29] H. C. Slider, Worldwide practical petroleum reservoir engineering methods. PennWell Books, 1983.

[30] Z. Chen, Reservoir simulation: mathematical techniques in oil recovery. Calgary, 2007: SIAM, 2007.

[31] J. Sheldon and W. Cardwell Jr, "One-dimensional, incompressible, noncapillary, two-phase fluid flow in a porous medium," T SPE AIME, no. 216, pp. 290“C296, 1959.

[32] H. L. Stone and A. O. Gardner, "Analysis of gas cap or dissolved gas reservoirs," T SPE AIME, no. 222, pp. 92 ${ }^{\circ} \mathrm{C} 104,1961$.

[33] A. Satter and G. M. Iqbal, Reservoir Engineering. Amsterdan, 2016: Elsevier - Gulf Publishing Company, 2016.

\section{Biography}

Antonio Fernando Britto is researcher at Centro Universitário SENAI-Cimatec. He holds $\mathrm{PhD}$ degree in fluid dynamics at SENAI-Cimatec, Brazil (2020). Previously he worked as an engineer in many renowned companies like Braskem, Petrobras, Vale, and Fluor Enterprises. He holds BS degree in chemical engineering from Universidade Federal da Bahia, MS degree from Universidade Federal de Pernambuco. His research interests include oil reservoir simulation, computational fluid dynamics (CFD) and developments in OpenFOAM.

Ivan Costa da Cunha Lima is Professor of the Postgraduate Program in Computational Modeling in Industrial Technology Centro Universitário SENAI-Cimatec. He holds $\mathrm{PhD}$ degree in physics at Universidade Estadual de Campinas, Uicamp, Brasil (1976), MS degree in Physis at Universidade Estadual de Campinas, Unicamp, Brasil (1972) and BS in Physics at Universidade Federal da Bahia (1968). Pos-doctorade in physics at Brown University (1983) and at Max-Planck Institute für Festkorperforschung (1977). His research interests include fluid dynamics, ocean engineering, fluid dynamic in porous media, and oil reservoir simulation. Da Cunha Lima has authored and/or coauthored more than 200 technical papers.

Andre Telles da Cunha Lima is Professor of the Postgraduate Program in Computational Modeling in Industrial Technology Centro Universitário SENAI-Cimatec and of the Universidade Federal da Bahia. He holds PhD degree in physics at Pontifícia Universidade Católica do Rio de Janeiro (2005), MS degree in Physics at Universidade do Estado do Rio de Janeiro (2000) and BS in physics at Universidade de São Paulo (1997). His research interests include fluid dynamics, ocean engineering, fluid dynamic in porous media, and oil reservoir simulation. Da Cunha Lima has authored and/or coauthored more than 35 technical papers. 